\title{
Cooperative Aquatic Sensing Using the Telesupervised Adaptive Ocean Sensor Fleet
}

\author{
John M. Dolan ${ }^{\mathrm{a}, \mathrm{b}}$, Gregg W. Podnar ${ }^{\mathrm{a}}$, Stephen Stancliff ${ }^{\mathrm{a}}$, Kian Hsiang Low ${ }^{\mathrm{b}}$, Alberto Elfes ${ }^{\mathrm{c}}$, John \\ Higinbotham $^{\mathrm{d}}$, Jeffrey Hosler ${ }^{\mathrm{e}}$, Tiffany Moisan ${ }^{\mathrm{f}}, \mathrm{John}_{\text {Moisan }}^{\mathrm{f}}$ \\ ${ }^{\mathrm{a}}$ The Robotics Institute, 5000 Forbes Avenue, Carnegie Mellon University, Pittsburgh, PA 15213 \\ ${ }^{\mathrm{b}}$ Dept. of Electrical and Computer Engineering, Carnegie Mellon University \\ ${ }^{\mathrm{c}}$ Jet Propulsion Laboratory, 4800 Oakgrove Drive, Pasadena, CA 91109 \\ ${ }^{\mathrm{d}}$ Emergent Space Technologies, Inc., Greenbelt, MD 20770 \\ ${ }^{\mathrm{e}}$ NASA Goddard Space Flight Center (GSFC), Greenbelt, MD 20771 \\ f NASA/GSFC Wallops Flight Facility, Wallops Island, VA 23337
}

\begin{abstract}
Earth science research must bridge the gap between the atmosphere and the ocean to foster understanding of Earth's climate and ecology. Typical ocean sensing is done with satellites or in situ buoys and research ships which are slow to reposition. Cloud cover inhibits study of localized transient phenomena such as Harmful Algal Blooms (HAB). A fleet of extended-deployment surface autonomous vehicles will enable in situ study of characteristics of HAB, coastal pollutants, and related phenomena. We have developed a multiplatform telesupervision architecture that supports adaptive reconfiguration based on environmental sensor inputs. Our system allows the autonomous repositioning of smart sensors for HAB study by networking a fleet of NOAA OASIS (Ocean Atmosphere Sensor Integration System) surface autonomous vehicles. In situ measurements intelligently modify the search for areas of high concentration. Inference Grid and complementary information-theoretic techniques support sensor fusion and analysis. Telesupervision supports sliding autonomy from high-level mission tasking, through vehicle and data monitoring, to teleoperation when direct human interaction is appropriate. This paper reports on experimental results from multi-platform tests conducted in the Chesapeake Bay and in Pittsburgh, Pennsylvania waters using OASIS platforms, autonomous kayaks, and multiple simulated platforms to conduct cooperative sensing of chlorophyll-a and water quality.
\end{abstract}

Keywords: harmful algal blooms, HAB, sensor web, ocean sensing, OASIS, adaptive sampling, telesupervision, multirobot systems

\section{INTRODUCTION}

Earth and ocean science research use data obtained from space, the atmosphere, the land, surface water, and the ocean to improve understanding of the Earth and its natural processes. Developing better models of ocean processes is crucial for meteorological and ecological studies, while surface water quality data yield insight into the suitability of the water for human use. Both are necessary for assessing water's ability to support aquatic life. At least three in every four humans depend on "surface water as their primary source of drinking water" ${ }^{1}$. Water resources in a region are critical to economic growth and quality of life.

Freshwater sensing is typically done with fixed sensors near tributaries, using periodic sampling. Ocean sensing is typically done with satellites, buoys, and crewed research vessels. Satellites are limited by cloud cover, temporal and geographical coverage, and resolution. Manned research vessels are expensive to deploy, and fixed sensors and anchored buoys cannot be easily moved to cover larger areas or to monitor regions of increased interest. Deploying mobile sensor platforms to augment fixed sensors and sensor buoys will help us better understand and model how contaminants/pollutants move and affect our environment, from industrial chemical and oil spills, to Harmful Algal Blooms (HABs). These platforms will also enable in situ meteorological studies of the atmosphere/ocean interface for hurricane prediction.

\footnotetext{
1 National Research Council. Earth Science and Applications from Space: National Imperatives for the Next Decade and Beyond, 2007.
} 
This paper describes experiments performed by a fleet of autonomous surface vehicles under the control of a multi-robot science exploration software architecture and system called Multilevel Autonomy Robot Telesupervision Architecture (MARTA) [1]. MARTA was initially developed for supervisory control of multiple robot assets exploring the lunar surface [2]. It has subsequently been expanded and instantiated in a system called TAOSF (Telesupervised Adaptive Ocean Sensor Fleet) for coordination and control of multiple robot boat exploration vehicles for oceanic [3] and freshwater research [4]. For the ocean boats, we have selected as an application the characterization of HABs, while for the freshwater vehicles, we are concentrating on their use in water quality studies.

In Section 2, we describe the autonomous aquatic platforms in greater detail, in Section 3 our science goals, and in Section 4 multiple experiments and associated results. Section 5 concludes with a summary of next steps in the development of TAOSF.

\section{AUTONOMOUS AQUATIC PLATFORMS}

\subsection{OASIS Ocean Platforms}

The OASIS (Ocean-Atmosphere Sensor Integration System) vessels are long-duration solar-powered autonomous surface vehicles (ASVs) designed for global open-ocean operations (Figure 1). Their development is funded by the National Oceanic and Atmospheric Administration (NOAA). The NOAA-funded OASIS Platform Build Team, which consists of EG\&G, Zinger Enterprises, and Emergent Space Technologies, provides vehicle development, payload integration and testing, operations, and maintenance of the OASIS fleet and ground systems.

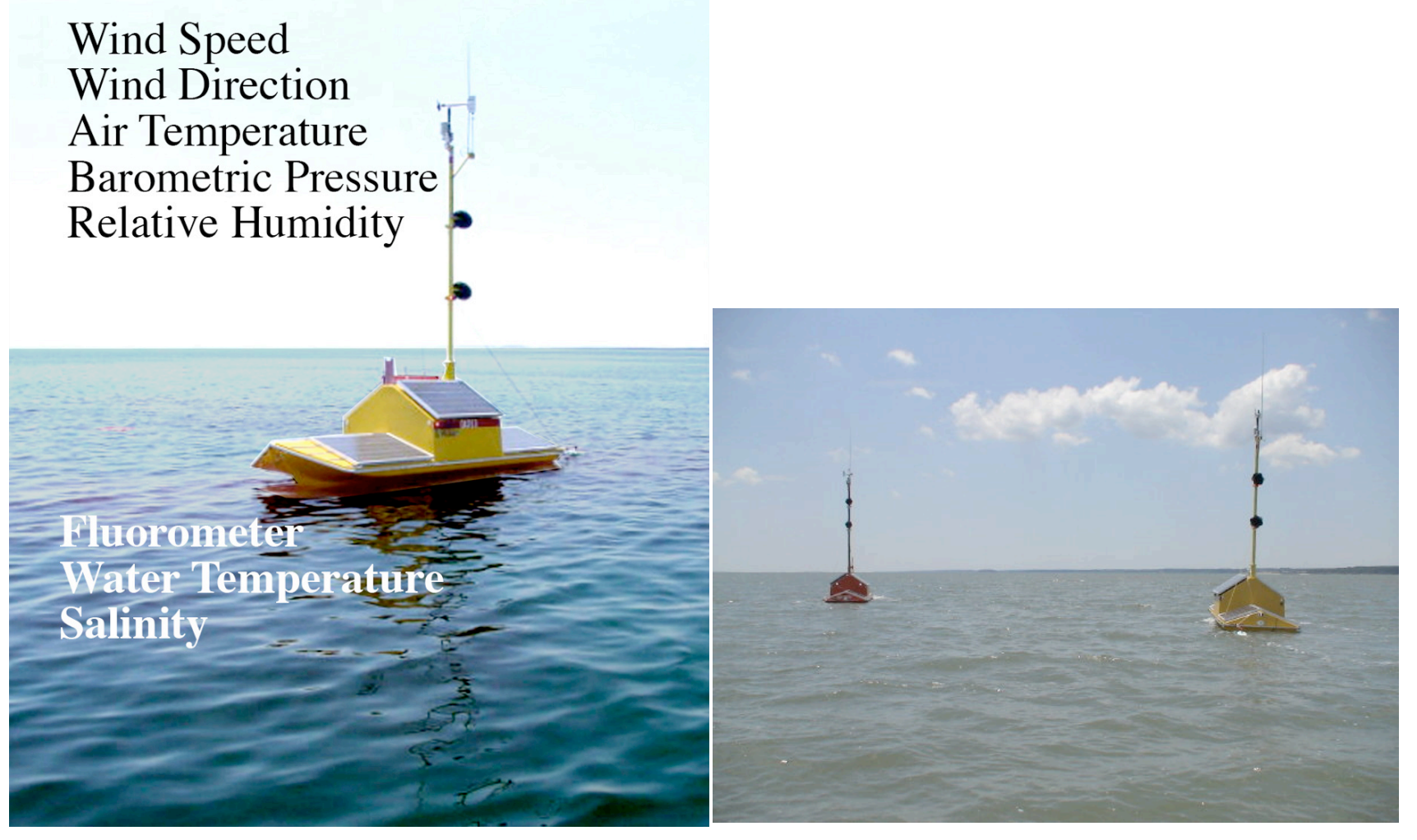

Figure 1. Left: OASIS platform with atmospheric and sea sensors identified. Right: Two OASIS platforms performing rhodamine WT dye-mapping in Chincoteague Bay, Virginia.

The OASIS platform is approximately 18 feet long and weighs just over $3000 \mathrm{lbs}$. The vehicle has a payload capacity of $500 \mathrm{lbs}$, and is designed to be self-righting to ensure survivability in heavy seas. It supports a wide range of communication links, including spread-spectrum radio, a cellular phone link, and an Iridium satellite link. 
Three platforms (named OASIS-1, OASIS-2, and OASIS-3) are operated by the NASA Wallops Flight Facility (WFF) on Chincoteague Island, Virginia and support operations for the TAOSF project. OASIS shakedown operations have been performed since early 2005 in the waters of the DELMARVA region, including the Chincoteague Bay and Pocomoke Sound. The first open-ocean deployment of the OASIS system was performed in November 2006. During this operation, the OASIS-2 platform successfully navigated over 8 nautical miles on a transect line established in the Atlantic Ocean off the coast from WFF.

Sensors onboard the OASIS platforms enable the collection of water salinity and conductivity data, sea surface temperature, and chlorophyll measurements. A rhodamine fluorometer was integrated to support mapping operations during TAOSF dye deployment tests. Dye is deployed to simulate HABs to allow development and testing of navigation and mapping algorithms. The forward payload bay provides space for installation of additional sensors. This bay includes a water flow-through system with manifolds and a de-bubbling system that simplifies installation of new sensors.

A mast-mounted meteorological station allows acquisition of atmospheric measurements, including barometric pressure, air temperature, relative humidity, wind speed, and wind direction. OASIS is also equipped with a forward-looking digital imaging system providing remotely located scientists with images of atmospheric and sea state conditions.

The off-board infrastructure developed by EST is known as the OASIS Mission Operations Environment (MOE). The MOE resides in the Wallops Coastal Ocean Observation Laboratory (WaCOOL) control room and provides applications and services that enable the WFF engineering and science operations team to perform platform commanding and telemetry monitoring, as well as communications management. The MOE also provides a middleware interface to enable other customers, such as the MARTA/TAOSF project, to integrate new systems that further enhance OASIS science operations.

In the current deployment configuration, both engineering telemetry (e.g., GPS position, roll, pitch, yaw, battery voltage) and science sensor data are communicated between each robotic platform and NASA's Goddard Space Flight Center via the Internet. It is from this distribution point that NOAA weather researchers will receive ocean and ocean/atmosphere interface sensor data.

\subsection{Robot Sensor Boats (RSBs)}

For the mobile deployment of freshwater quality sensors, a fleet of small and relatively inexpensive Robotic Sensor Boats (RSBs) is being developed at CMU (Figure 2). The design is based on commercially available components such as the hull, drive system, and communications system. Customization is kept to a minimum with the primary focus being kept on the sensing and navigation requirements.
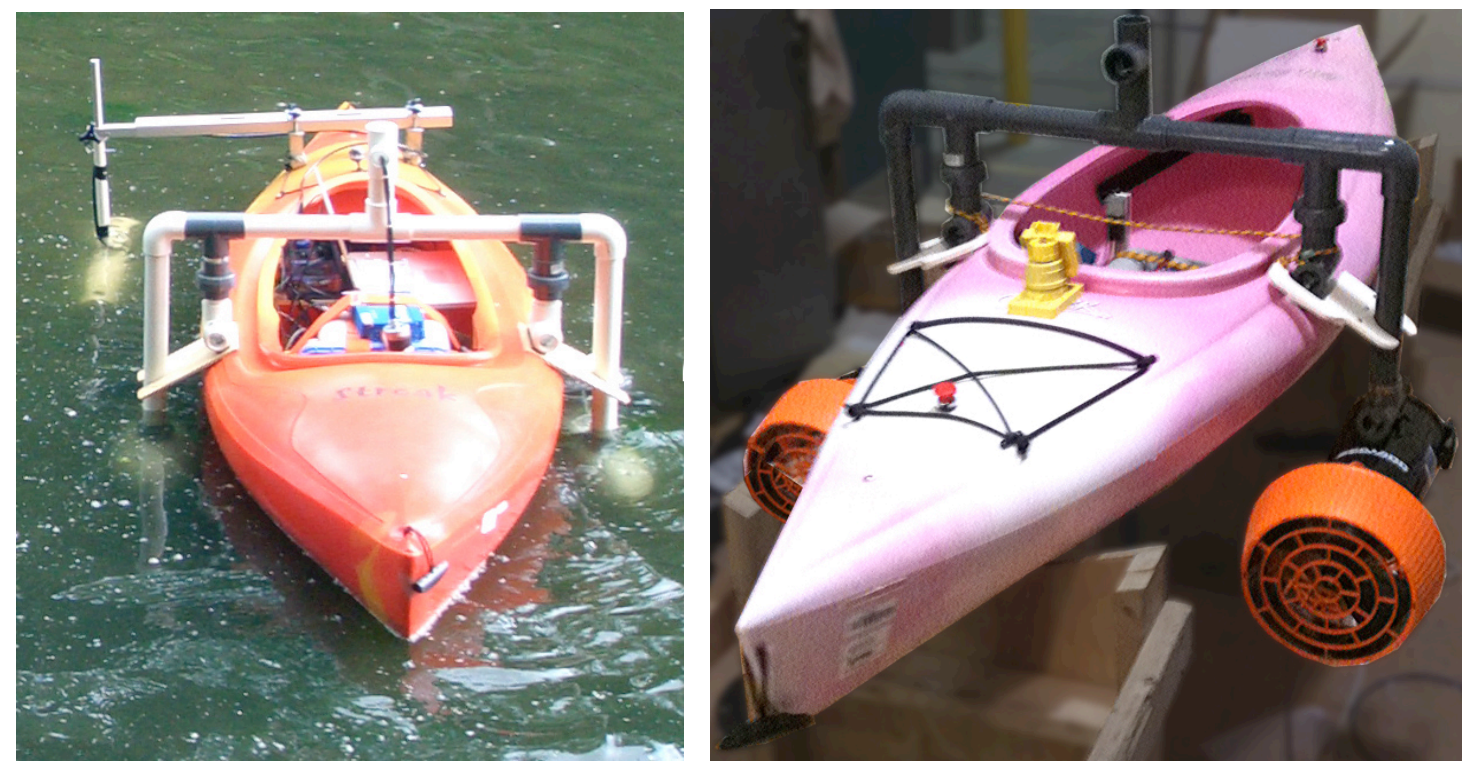

Figure 2. Left: RSB-1 showing water quality sonde housing on the port bow (under water). Right: RSB-2 nearing completion. 
The hull is a relatively inexpensive roto-molded recreational kayak that is fitted with a ducted thruster on each side. The thrusters are based on personal SCUBA propulsion units with screens to exclude foreign materials and to provide safety to humans operating in the same area. The dual thrusters allow simple differential steering and provide adequate speed when deploying each boat to its sampling area. A package of telemetry and water quality sensors is supported. A sealed spiral-cell deep-cycle marine battery provides power adequate for runs of many hours and also acts as ballast. Telemetry and science data, as well as control signals are all handled over a cellular modem.

\section{SCIENCE GOALS: HARMFUL ALGAL BLOOMS AND WATER QUALITY SENSING}

Interest in Harmful Algal Bloom (HAB) detection has grown in recent years for scientific, commercial public health reasons. Depending on the type of algae present, HABs have been shown to be dangerous to sea life and to human health. There is a significant interest in identifying environmental factors that contribute to the occurrence of HABs, so that these may be incorporated in bloom prediction algorithms. These factors may include time of year, salinity, and seasurface temperature to predict the abundance (low, medium, or high) and type of HABs. Since HABs are sporadic ocean phenomena, we used first rhodamine WT (water-tracing) dye as a HAB surrogate, then more recently chlorophyll-a.

The TAOSF instantiation of MARTA will provide the following advantages over existing systems for observing and analyzing HABs: 1) Dynamic tasking and adaptation; 2) Higher in situ resolution and greater insensitivity to cloud cover in comparison to current satellite systems; 3) Access to and greater agility in coastal waters than what is available through buoys; 4) Real-time multipoint science data observations and generation of associated interpretations by remotely located oceanographers.
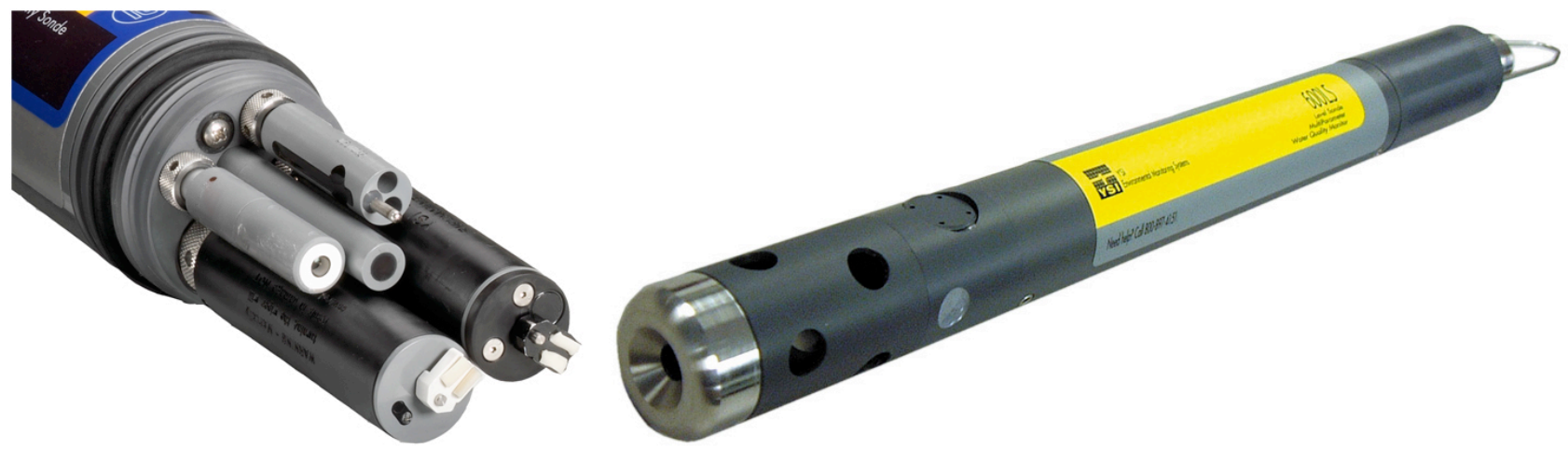

Figure 3. Left: A YSI water quality sonde showing multiple sensor probes. Right: YSI sonde similar to the one deployed by RSB-1

For investigating an algal bloom, researchers at Carnegie Mellon University take control of the OASIS platforms and provide high-level planning and monitoring. The OASIS collection of weather-related data mentioned earlier is not interrupted, but the additional sensing for investigating the algal bloom is brought online to map the extent of a bloom, chlorophyll concentrations, and with additional sensors, eventually give a shore-based biologist the ability to assess whether the algal species are harmful. If they are, local authorities can be notified at aquaculture businesses, fisheries, and beaches.

For investigating water quality, the YSI 600R multi-parameter sonde deployed by the RSB-1 includes multiple sensor probes for temperature, $\mathrm{pH}$, dissolved oxygen, and conductivity (see Figure 3 ).

\section{EXPERIMENTAL RESULTS}

This section summarizes results from field tests conducted during the summers of 2007-2009. The 2007 [3] and 2008 [5] tests are described in greater detail in previous publications, but are summarized here to give background information. All OASIS tests were conducted off Greenbackville, Virginia in Chincoteague Bay on the Atlantic Ocean side of the Chesapeake Peninsula. The Robot Sensor Boat tests were conducted in Panther Hollow Lake, an artificial lake in Schenley Park near Carnegie Mellon University’s campus in Pittsburgh, Pennsylvania. 


\subsection{OASIS single-platform dye mapping without drift compensation (August 2007)}

In this test, we first laid down a patch of rhodamine dye about $120 \mathrm{~m}$ in length and $30 \mathrm{~m}$ in width (see Figure 4 , left). A single OASIS platform equipped with a fluorometer capable of sensing the dye then performed a spiral pattern, the default provided by the Adaptive Sensor Fleet software that NASA Goddard contributed to the project. An aerostat tethered to a manned tender boat carried an instrument package aloft including GPS, altimeter, compass, and video camera to provide validation images of the data gathered by the OASIS platform. We used a discretized version of Markov Random Fields, called a spatio-temporal Markov Random Lattice (ST-MRL), to encode the data obtained by the OASIS platform [6]. Associated with the ST-MRL we maintain additional stochastic lattice-based layers for inference and decision, which are used to plan and control the activities of the platform [7]. The structure that incorporates both the ST-MRL and the information-theoretic inference and decision layers is called an Inference Grid (IG). The rhodamine dye concentration measurements taken by the OASIS platforms during field tests are used as input to the Inference Grid mapping process, with results shown in Figure 5. The fluorometer measurements were used to compute the presence or absence of dye for each cell in the area traversed by an OASIS boat.
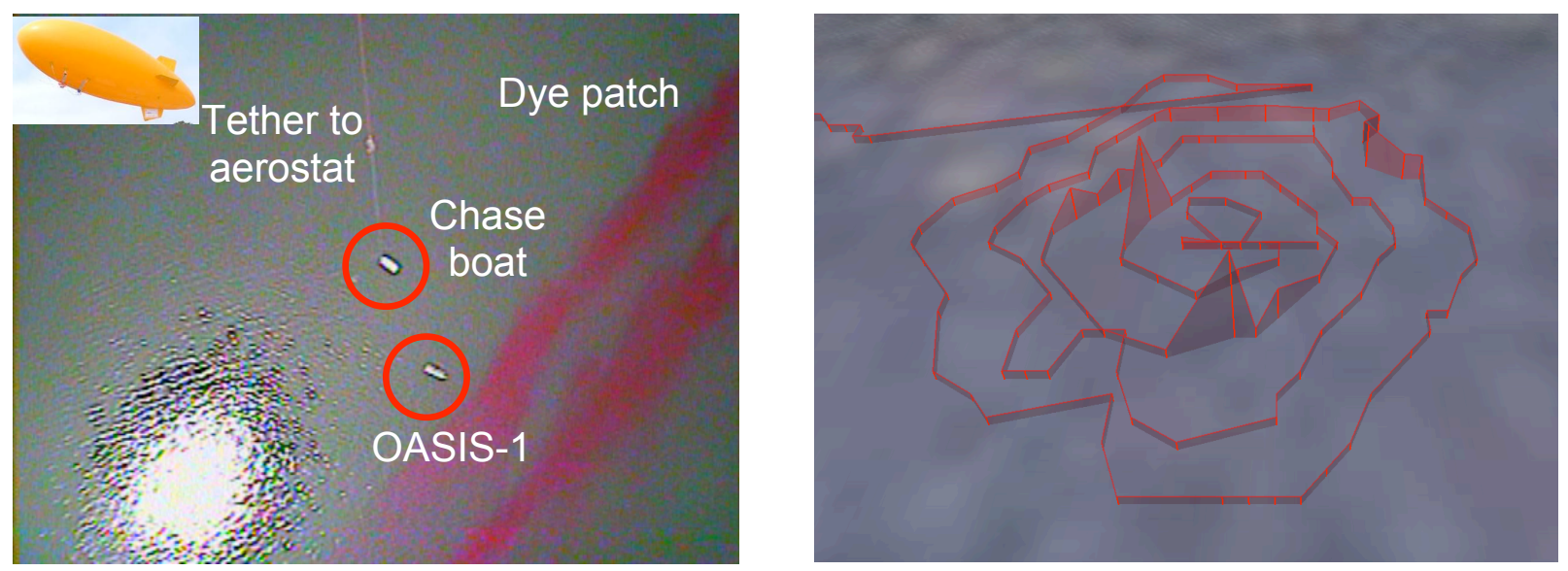

Figure 4. Left: Aerial view from the aerostat observation platform. The chase boat (with aerostat tether) and OASIS-1 platforms are circled in red. The image comes from the aerostat itself, which is depicted in the upper-left inset. The parallel red dye stripes serving as surrogate HABs are also visible. Right: A slanted view of the ocean area over which a spiral search was conducted by OASIS-1. The measured rhodamine concentrations are shown as a vertical "ribbon" at each sampling point.
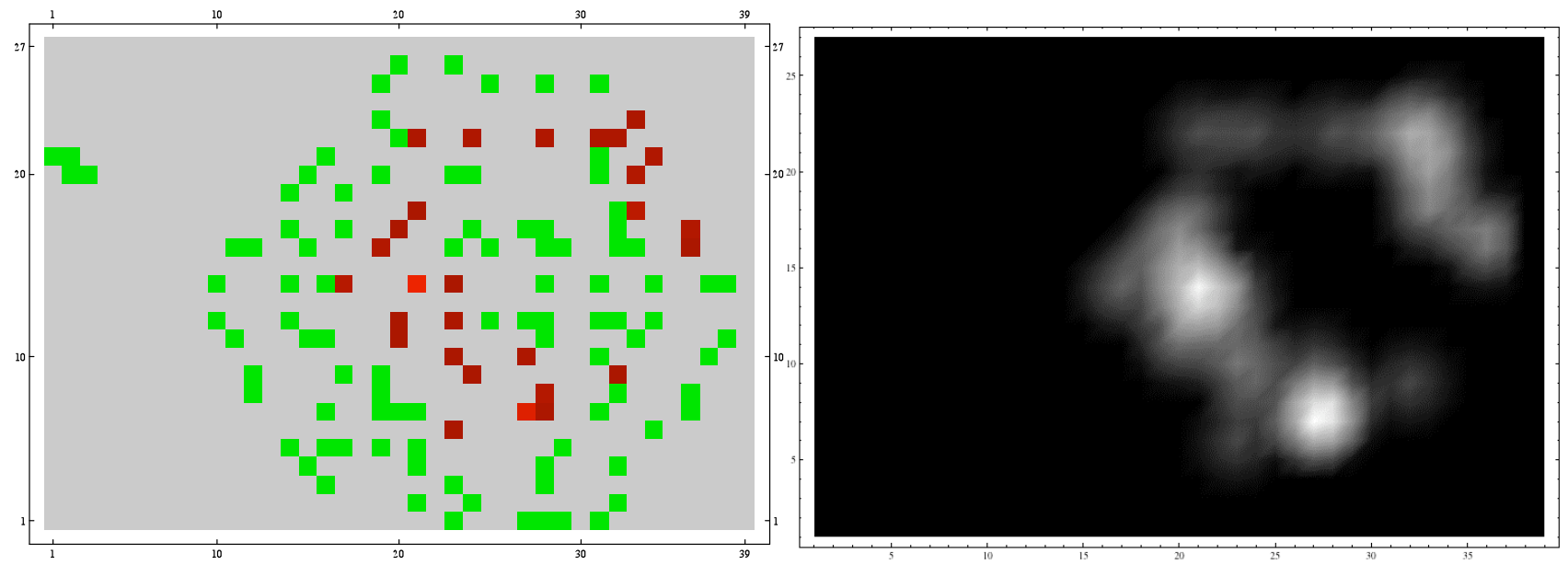

Figure 5. August 2007 test Inference Grid plots with $\mathrm{x}$ - and y-axes in meters. Left: This plot shows the areas with high probability of dye presence (in red), high probability of dye absence (in green), and high entropy or lack of information (in grey). Right: This plot shows inferred hypotheses of algal blooms (dye tracks), where white corresponds to high probability of a bloom and black to no measurement. The areas of high entropy can be used to replan the search pattern of the boat. 


\subsection{OASIS multi-platform dye mapping with drift compensation (July 2008)}

In the August 2007 test, the number of dye samples the OASIS-1 platform was able to take was limited by the fact that the water current caused the dye to drift, and the spiral pattern caused the platform to spend much of the time away from the dye patch. In our July 2008 field test, we addressed the first problem by implementing "drift compensation", i.e., warping the platform's GPS waypoints by their expected drift (i.e., the current vector multiplied by time) such that they map out a desired path in water-surface, rather than GPS, coordinates. In the degenerate case of zero current, the watersurface and GPS coordinates are the same. We addressed the second problem by using lawnmower and box patterns for the two OASIS platforms involved in this test, depicted in Figure 6. A drift buoy instrumented with GPS was used to measure the current. Two rhodamine WT dye stripes were then laid down parallel to the current. OASIS-2 (Boat A) followed a lawnmower pattern which progressed in the current direction along the dye stripes. To achieve the depicted rectilinear pattern in water-surface coordinates, its GPS coordinates formed a trapezoidal pattern, with diagonal long legs and elongated short legs. OASIS-3 (Boat B) followed a rectangular box pattern in GPS coordinates such that the current carried the stripes through the box.

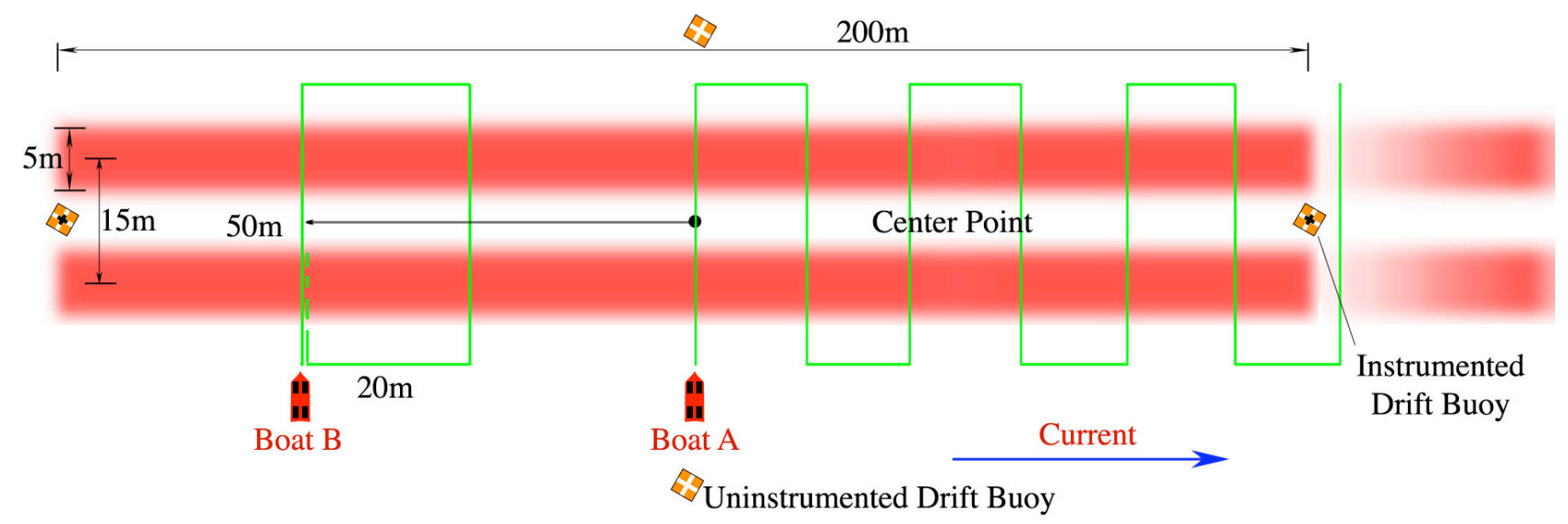

Figure 6. July 2008 multi-OASIS platform field test concept. An instrumented drift buoy provides a estimate of the water current. Boat A performs a drift-compensated lawnmower pattern in order to keep the dye stripes from "running away". Boat B performs a rectangular box pattern in GPS coordinates as the stripes drift through it. The goal is for the OASIS platforms jointly to map the dye.

Figure 7 shows the dye-stripe mapping results from the July 2008 test. An inter-dye-stripe gap is intuitively clear from the left-side plot of the two OASIS platform trajectories and their sample points exceeding a threshold value corresponding to dye presence. Because the sampling is sparse, a full map is inferred in the right-side plots using a nonparametric probabilistic inference model called log-Gaussian which is described in greater detail in [8]. The character of the resultant inferred stripes corresponds to that expected from the dye laid down according to Figure 6. 

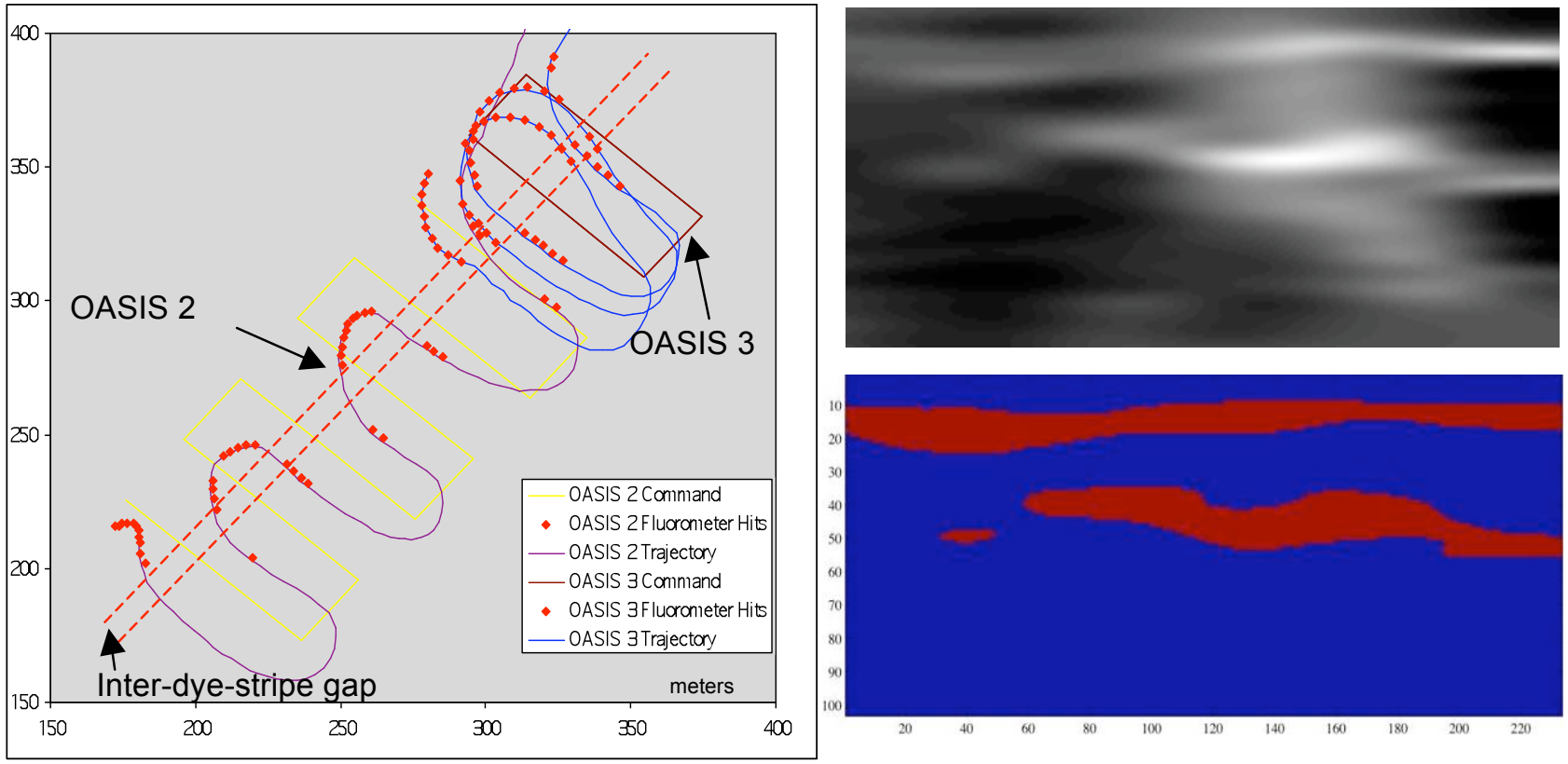

Figure 7. July 2008 test samples (left) and inferred dye maps (right). Left: Platform trajectories are shown in driftcompensated water-surface coordinates. The yellow and purple paths are respectively OASIS-2's commanded lawnmower pattern and actual path, while the brown and blue paths are respectively OASIS-3's commanded box pattern and actual path. The red sample points correspond to a fluorometer reading above a threshold value. An inter-dye-stripe gap can be seen, with some points in the upper right erroneously in the gap due probably to drift compensation error and/or dye stripe diffusion. Right upper: Grey-scale log-Gaussian map inference of rhodamine dye concentration. Right lower: Thresholding of the right upper plot to produce binary classification. The horizontal axis of the right-side plots corresponds to the interdye-stripe gap axis in the left-side plot.

\subsection{June 2009 OASIS multi-platform chlorophyll-a test}

In this test, we began to sense chlorophyll-a (chl-a) as a surrogate for HABs. Chl-a is present in many types of algae, and is capable of detection via the fluorometers with which the OASIS platforms are equipped. The primary objective of this test was to characterize and validate the OASIS platforms' ability to map concentrations of chl-a over a significant area with two OASIS platforms and using a variety of mapping patterns. For each test, each platform was commanded to execute the same pattern with a small temporal offset so that one would effectively follow the other, sampling the same surface region and allowing cross-validation of results. The autonomous platforms were followed in turn by a field team taking water samples from the same area to be analyzed later for actual chl-a concentrations, providing "ground truth" for the experiment.

Three lawnmower-pattern scan types were conducted: 1) a 250m x 250m north-south scan; 2) a 250m x 250m east-west scan (Figure 8, left); and 3) a 250m x 500m north-south scan with the longer dimension in the north-south direction (Figure 8, right). For scan types 1 and 2, the distance between legs was 25m; for scan type 3, it was 50m. Both northsouth (NS) and east-west (EW) scans start from the bottom lefthand, or southwest, corner. A total of four scans were conducted with each of the two OASIS platforms, two of type 1, and one each of types 2 and 3 . The times elapsed between the first platform's start and the second platform's start in the four scans ranged between 9 and 15 minutes. 

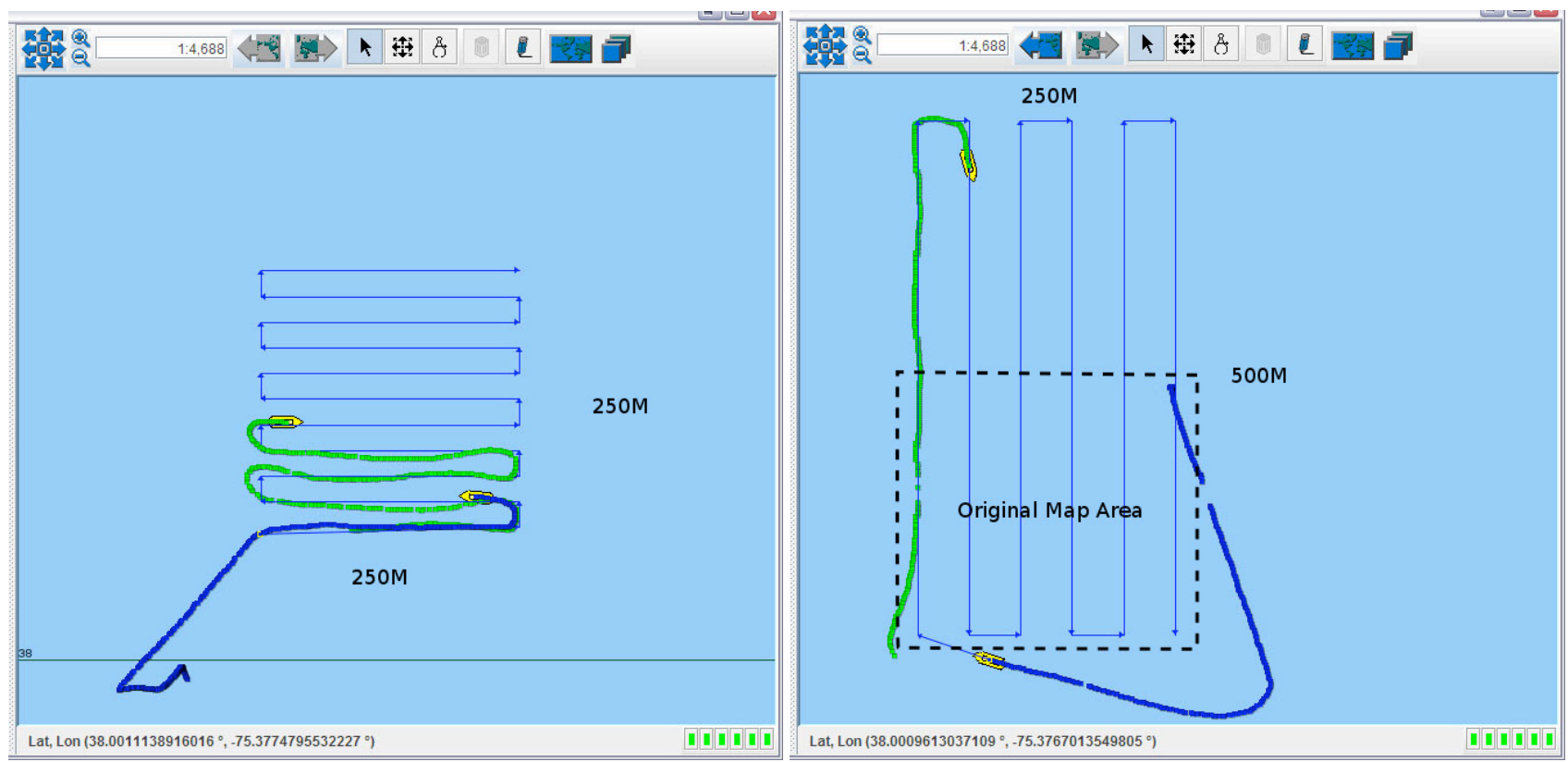

Figure 8. Type 2 and 3 scan patterns from the June 2009 OASIS chl-a mapping test. Left: The east-west scan (scan type 2). The north-south scan (scan type 1) covers the same area but is rotated $90^{\circ}$. The lead platform has completed four legs and is beginning the fifth, while the second platform has completed one leg and is beginning the second. Right: The elongated north-south scan (scan type 3). The original $250 \mathrm{~m} \times 250 \mathrm{~m}$ map area for scans 1 and 2 is the bottom half of this scan, shown inside the dotted lines. The lead platform has completed one leg and is beginning the second, while the second platform is about to begin the first leg.

A typical set of samples is shown in Figure 9 for a type-1 (north-south) and type-2 (east-west) scan. We applied Gaussian process-based inference to this sparse set of samples relative to the full map size (250m x 250m for types 1 and 2 and $250 \mathrm{~m} \times 500 \mathrm{~m}$ for type 3 ) in order to create the inferred maps shown in Figure X. In this figure, the lead OASIS platform's four scans are shown from left to right across the top, and the follow-on OASIS platform's four scans are shown from left to right across the bottom. The chl-a concentration in $\mathrm{mg} / \mathrm{m}^{3}$ is shown via the color coding, with red indicating high concentration and blue indicating low concentration. The plots are aligned and scaled so that the bottom left-hand corner (roughly $500 \mathrm{~m}, 200 \mathrm{~m}$ ) always refers to the same GPS coordinate, and the type- 4 scan is appropriately elongated in the y-direction. The total amount of time for the depicted scans was 4 hours, 19 minutes. The start times and durations of each of the eight scans are given in the figure.
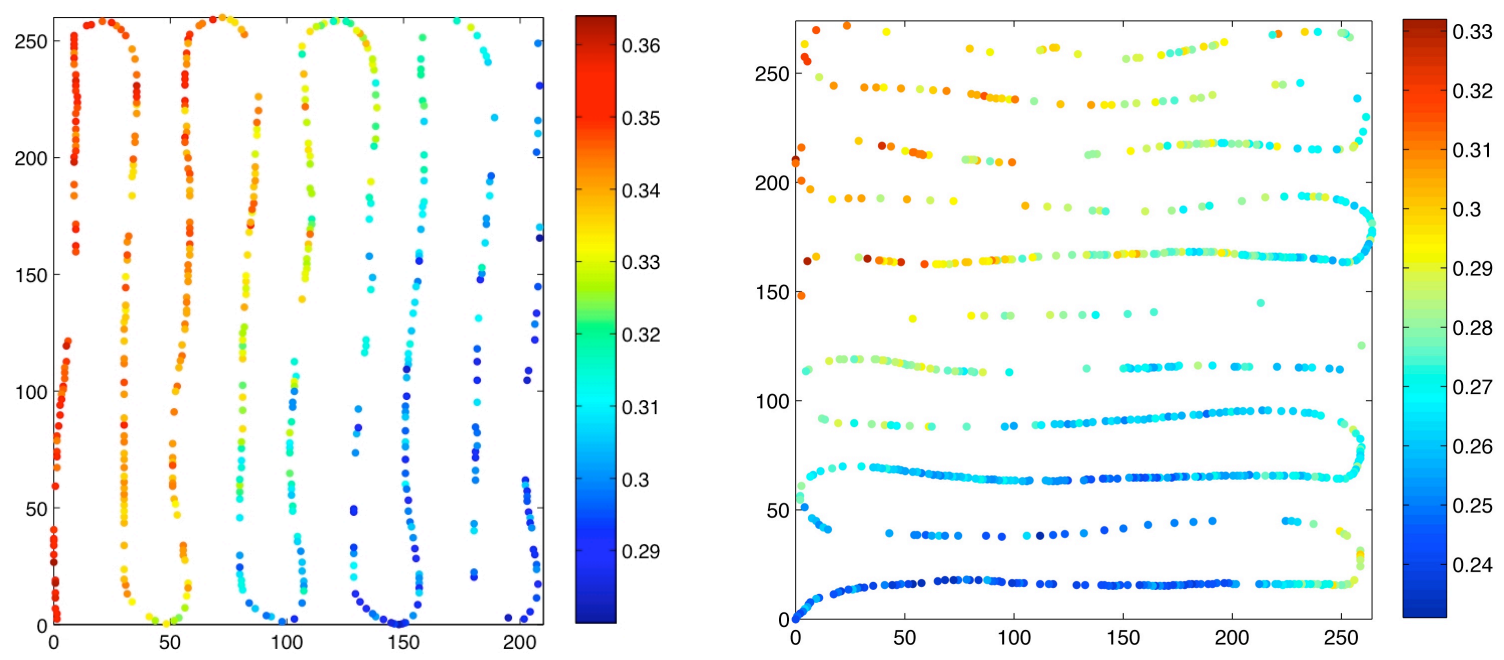

Figure 9. Left: Type-1 (north-south) scan. Right: Type-2 (east-west) scan. The axes are in meters, and the color coding shows the chl-a concentration in volts measured by the fluorometer. In Fig. 10, volts have been transformed to $\mathrm{mg} / \mathrm{m}^{3}$. 
The inferred maps in Figure 10 are generally consistent across the two OASIS platforms. For example, subplots 1 (Platform 1) and 5 (Platform 2) show a north-south front of high chl-a concentration that appears to be moving in a westward direction, since Platform 2's north-south boundary occurs further to the west than Platform 1's. In the second type-1 scan (subplots 2 and 6), begun 54 minutes after the first, the chl-a readings are significantly lower, and the highconcentration front appears to have continued to move through the patch to the west. In the type- 2 scans (subplots 3 and 7), the data from the two platforms are generally consistent, with a reduced concentration seen in Platform 2's upper lefthand corner that may correspond to a drift of a chl-a front in a northwesterly direction. The boundary between higher and lower concentration has shifted from a north-south in the type-1 scan to a southwest-to-northeast direction. In order to check whether this was due to a sensing artifact resulting from the main direction of the platform travel, we reverted for the fourth set of scans (subplots 4 and 8) to a north-south direction. The resultant maps are generally consistent with the type-2 scans and are also consistent with a northwesterly movement of the yellow higher-concentration chl-a mass.

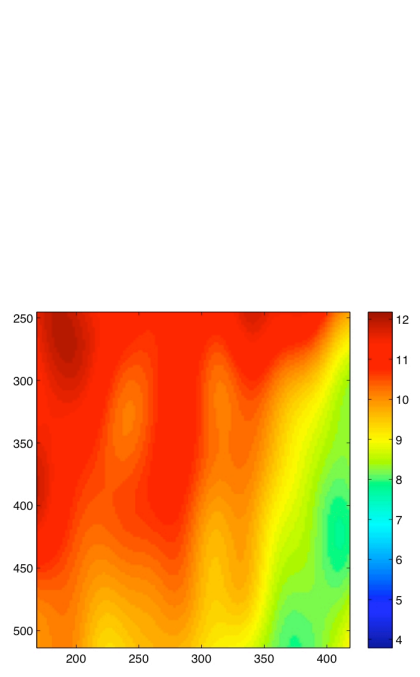

1) Scan type 1 (NS) \#1, Platform 1 Start: 0:00 Duration: 39:29

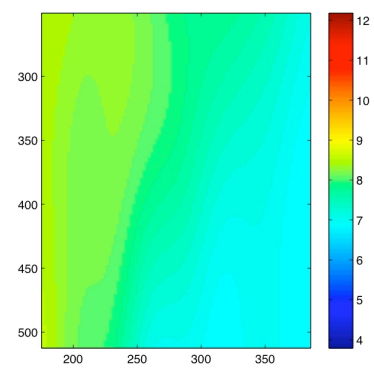

2) Scan type 1 (NS) \#2, Platform 1 Start: 54:21 Duration: 35:37

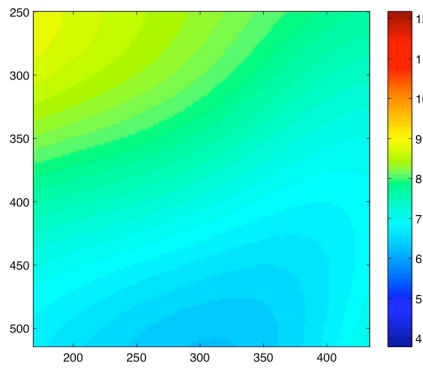

3) Scan type 2 (EW), Platform 1 Start: 2:08:26 Duration: 45:03

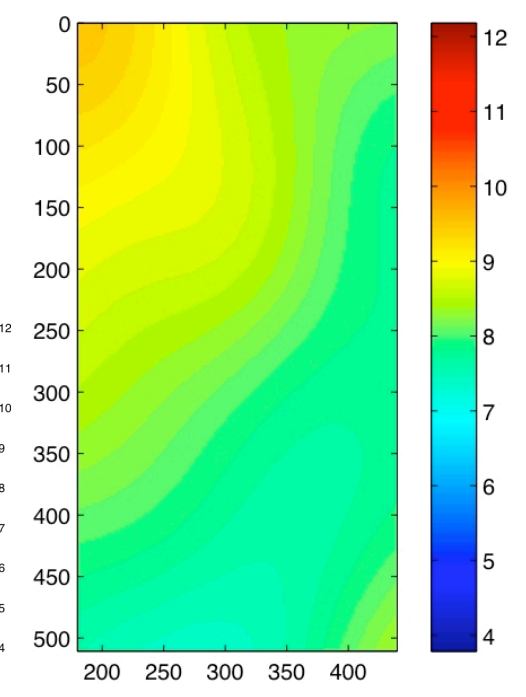

4) Scan type 3 (NS), Platform 1 Start: 3:14:22 Duration: 51:32

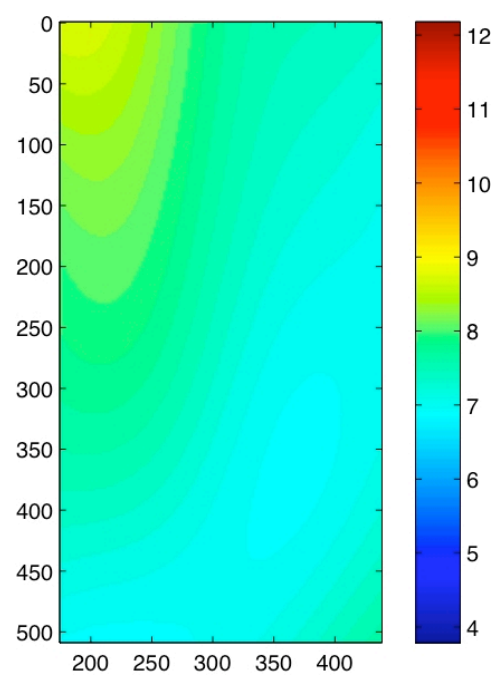

8) Scan type 3 (NS), Platform 2 Start: 3:23:30 Duration: 55:45

5) Scan type 1 (NS) \#1, Platform 2 6) Scan type 1 (NS) \#2, Platform 2 Start: 14:15 Duration: 24:09

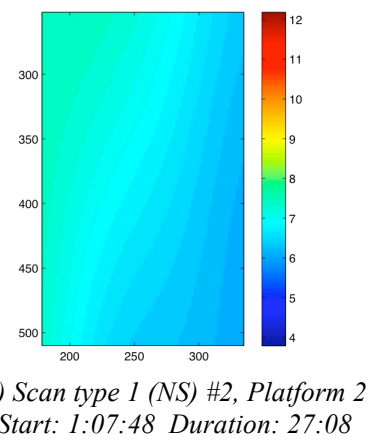

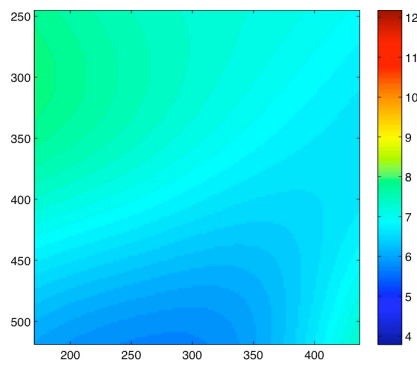

7) Scan type 2 (EW), Platform 2 Start: 2:19:21 Duration: 50:24

Figure 10. Inferred chl-a maps from two OASIS platforms scanning in Chincoteague Bay. The top row shows maps from Platform 1's data, moving left to right in time. Similarly, the bottom row shows maps from Platform 2's data. Start times and scan durations are shown, with all start times relative to the beginning of Platform 1's first scan. 


\subsection{Summer 2009 Robot Sensor Boat water quality tests}

In parallel with the OASIS HAB-oriented tests in the Chesapeake peninsula area, during 2009 we have conducted water quality tests using RSB-1 on Panther Hollow Lake, a man-made lake in Schenley Park, which adjoins Carnegie Mellon University in Pittsburgh, Pennsylvania. Figure 11 shows an aerial view of Panther Hollow Lake, with RSB-1 sample points from a June 16, 2009 test superimposed. Figure 12 shows views of RSB-1 operating in the lake.

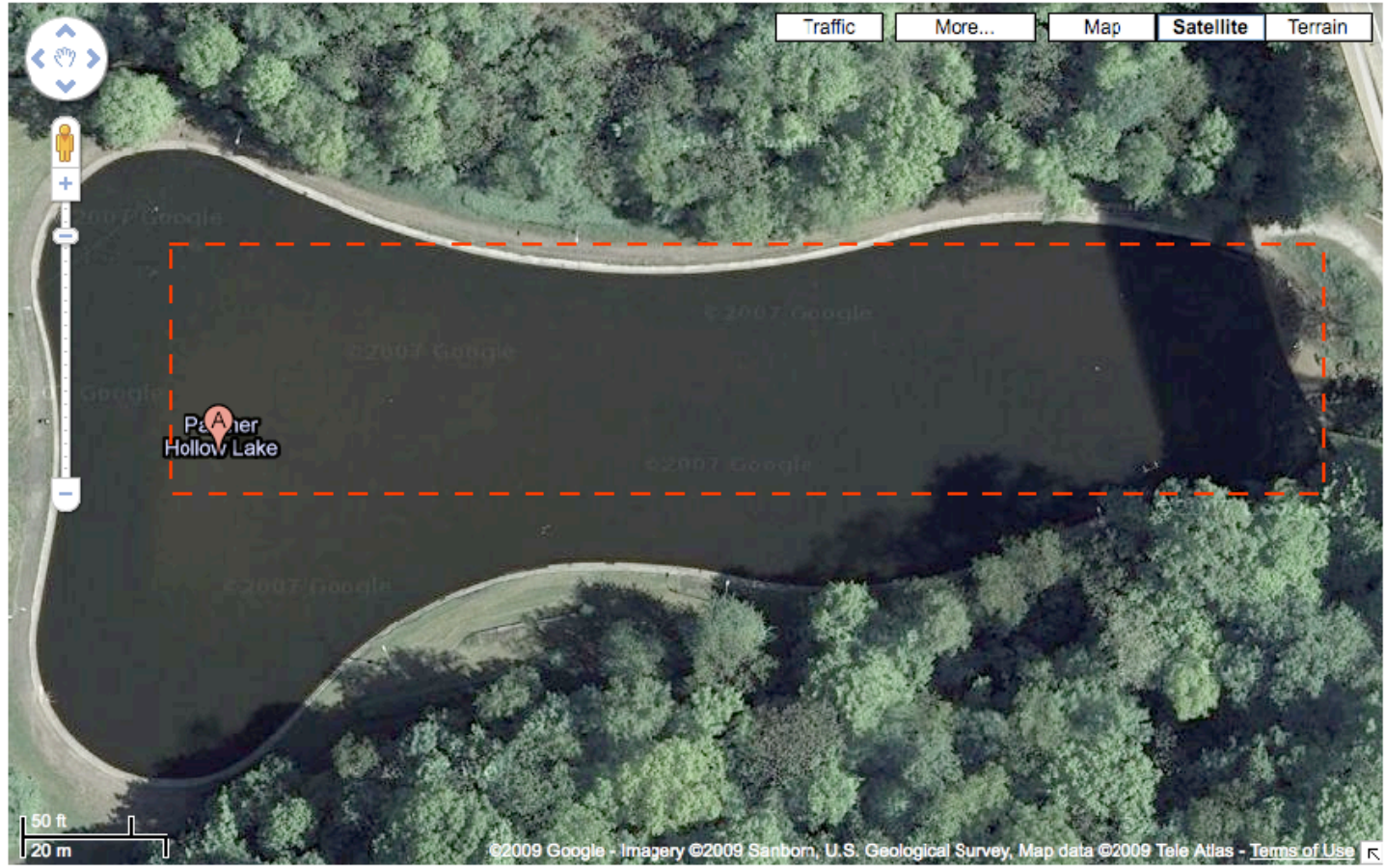

Figure 11. Aerial view of Panther Hollow Lake with the RSB-1 operating area from a May 29, 2009 test indicated.
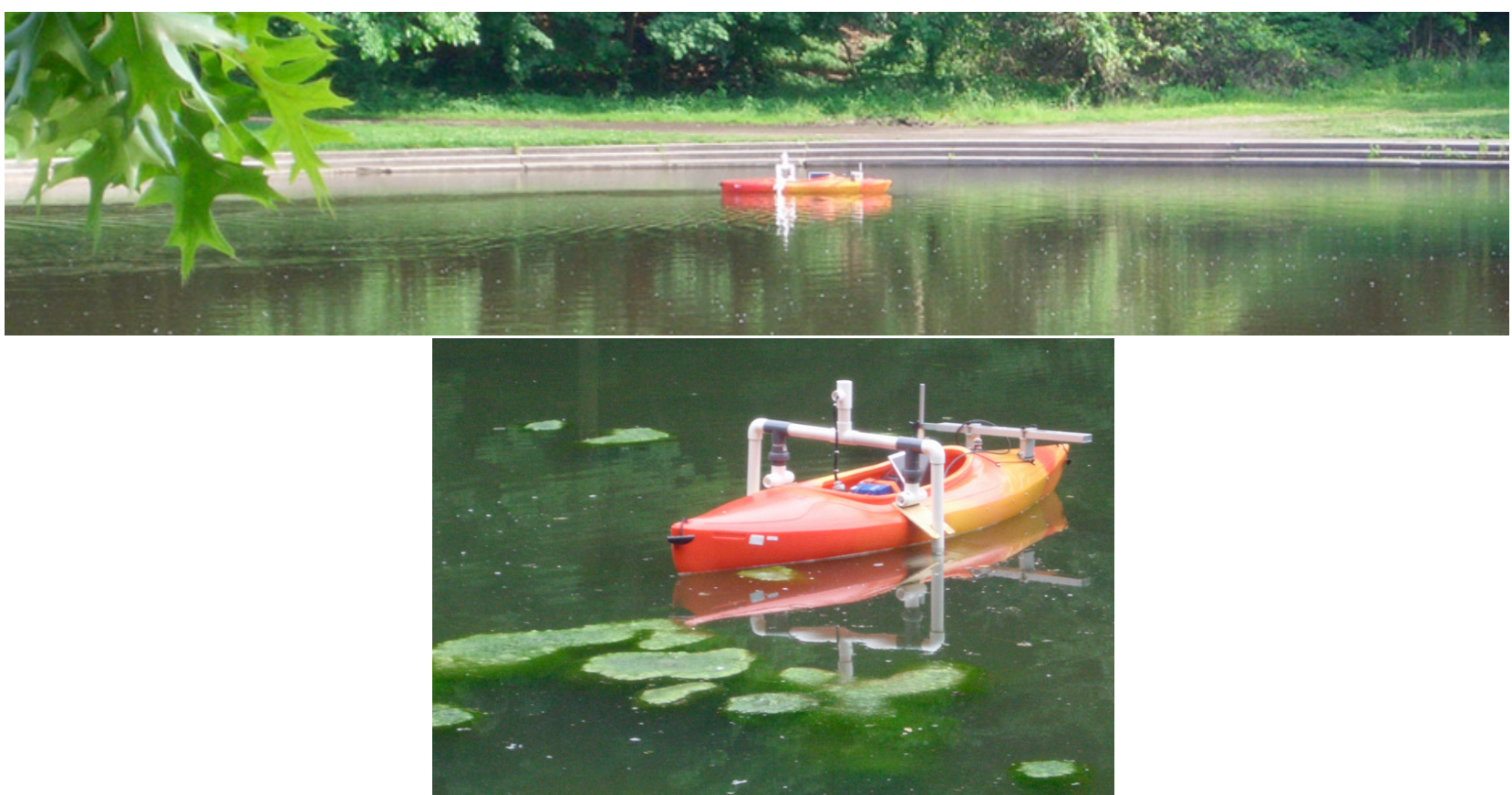

Figure 12. RSB-1 in Panther Hollow Lake under varying conditions. Top: Clear. Bottom: Significant "hair" algae present. 

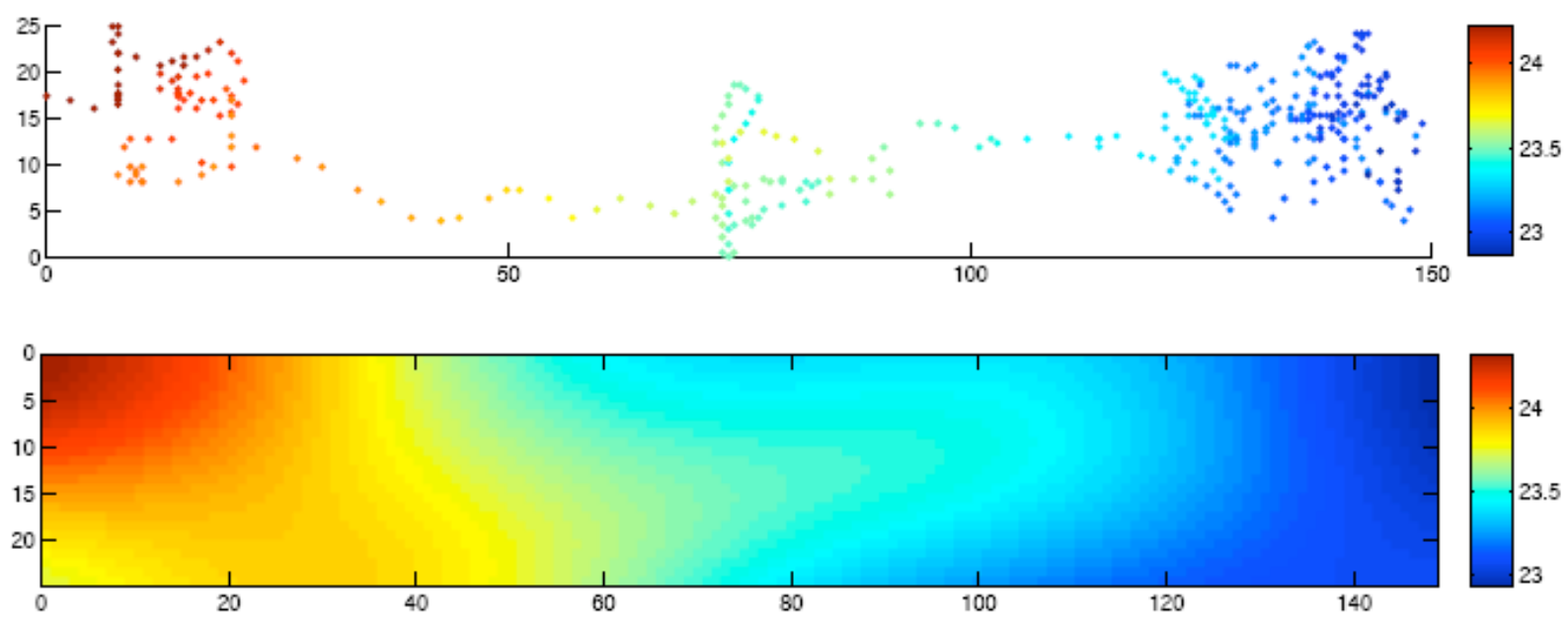

Figure 13. RSB-1 water sonde temperature data, degrees Celsius shown in color scale to right of each plot. The $\mathrm{x}$ and $\mathrm{y}$ axes are in meters. Top: RSB path and individual readings. Bottom: Gaussian process-based inference of the full temperature field.

Figure 13 shows RSB-1 water sonde sampled and inferred temperature field. Those for dissolved oxygen and $\mathrm{pH}$ were also acquired. The temperature and dissolved oxygen fields make intuitive sense given what we can observe independently about the lake. Specifically, the temperatures were higher to the left of the image and lower on the right, where a bridge casts a shadow. The high dissolved oxygen readings on the right are probably related to the presence at that end of the lake of a layer of algae. We have given the data from this initial and subsequent tests to Water Quest (http://www.ce.cmu.edu/ wquest/Education\%20and\%20Outreach/phl.htm), a project led by Prof. Jeanne Van Briesen in Carnegie Mellon University's Civil Engineering department that monitors water quality in Panther Hollow Lake in connection with a restoration of the lake planned by the Pittsburgh Parks Conservancy (http://www.pittsburghparks.org/).

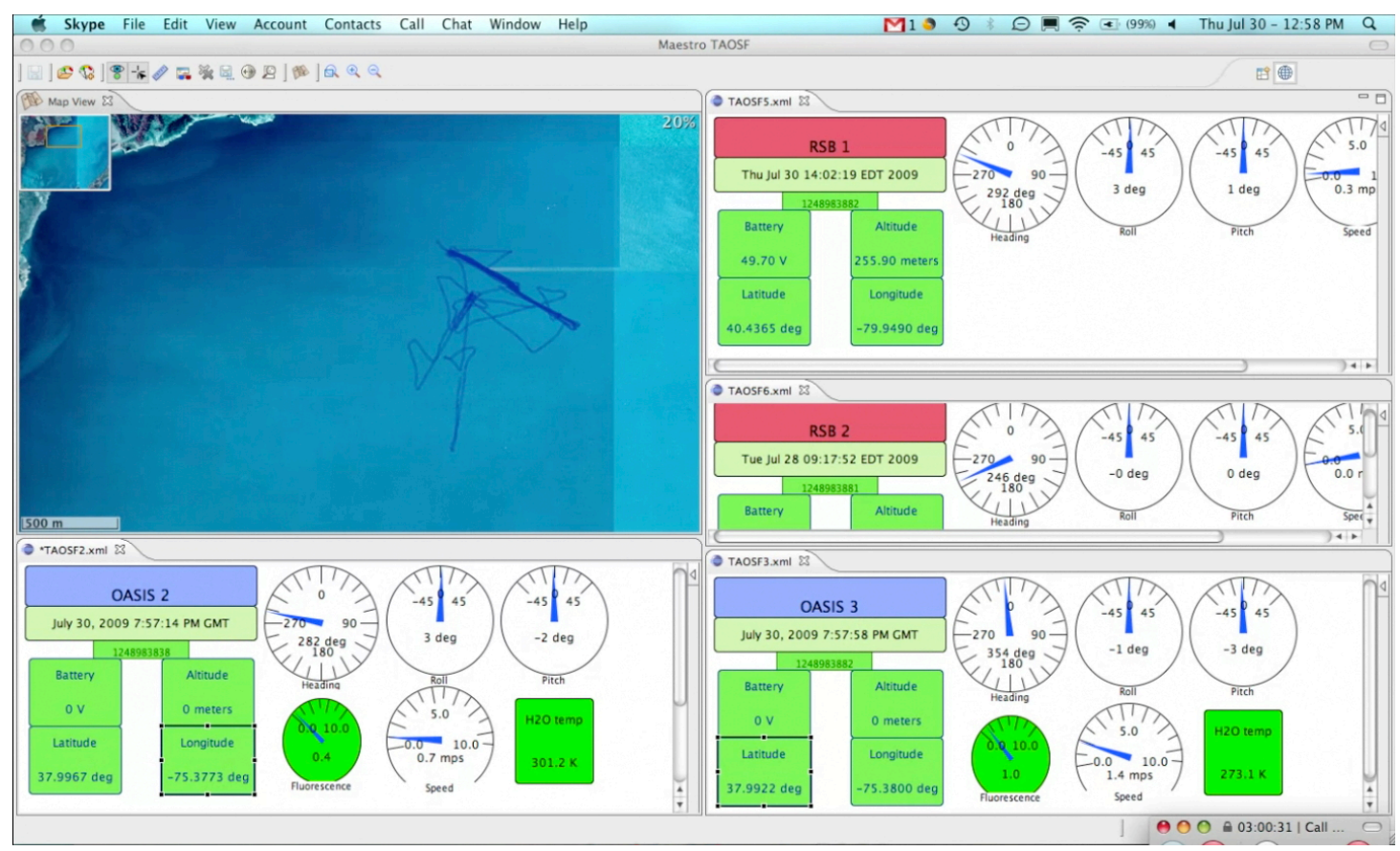

Figure 14. Platform and data visualization interface.

\subsection{Platform and data visualization}

Tests conducted in the last week of July 2009 involved the multiple operation of two OASIS platforms, two RSBs, and multiple simulated platforms within the TAOSF telesupervision architecture. There has not yet been time to analyze and 
present the data from these tests, but Figure 14 shows a platform and data visualization interface rapidly prototyped for use in these recent tests by the Jet Propulsion Laboratory members of our team based on the Maestro software system (http://www-robotics.jpl.nasa.gov/facilities/facility.cfm?Facility=9) that has been used extensively for collaborative activity planning for the Mars Exploration Rovers. The upper lefthand corner shows a map of the Virginia coastline near Greenbackville, and four "dashboards" with instruments and indicators are shown, two for the OASIS platforms and two for the RSBs.

\section{CONCLUSIONS}

This paper presents the Telesupervised Adaptive Ocean Sensor Fleet (TAOSF), an instantiation of the MARTA multilevel autonomy control architecture for investigation of HABs and water quality. Advantages over existing systems for observing and analyzing HABs include: dynamic tasking and adaptation; higher in situ resolution and greater insensitivity to cloud cover (as compared with satellite systems); access to and greater agility in coastal waters than that available through buoys; real-time multipoint science data observations and generation of associated interpretations by remotely located oceanographers. Collected data are made available to other scientists via the world-wide-web both as they are collected, and from an historical data archive server. The described experimental results give insight into chlorophyll-a formations and water quality patterns. Future tests will incorporate adaptive sensing and expand the number of platforms simultaneously telesupervised within the architecture.

\section{ACKNOWLEDGMENTS}

This work was supported by NASA award NNX06AF27G, "Telesupervised Adaptive Ocean Sensor Fleet", granted under the Advanced Information Systems Technology program of NASA's Earth Systems Technology Office (ESTO). The TAOSF project is a collaboration among Carnegie Mellon University (CMU), NASA Goddard Space Flight Center (GSFC), NASA Goddard's Wallops Flight Facility (WFF), Emergent Space Technologies, Inc. (EST), and the Jet Propulsion Laboratory (JPL). Work on the OASIS platforms is conducted by Emergent Space Technologies, Inc., EG\&G, and Zinger Enterprises under award NA03NOS4730220 from the National Oceanic and Atmospheric Administration (NOAA), U.S. Department of Commerce.

\section{REFERENCES}

[1] Podnar, G.W., Dolan, J.M., Elfes, A. and Bergerman, M., "Multi-level autonomy robot telesupervision," Proc. ICRA 2008 Workshop on New Vistas and Challenges in Telerobotics (2008).

[2] Halberstam, E., Navarro-Serment, L., Conescu, R., Mau, S., Podnar, G.W., Guisewite, A.D., Brown, H.B., Elfes, A., Dolan, J.M. and Bergerman, M., "A robot supervision architecture for safe and efficient space exploration and operation," Proc. 10th Biennial International Conf. on Engineering, Construction, and Operations in Challenging Environments (2006).

[3] Podnar, G.W., Dolan, J.M., Elfes, A., Stancliff, S., Lin, E., Hosler, J.C., Ames, T.J., Moisan, J.R., Moisan, T.A., Higinbotham, J. and Kulczycki, E.A., "Operation of robotic science boats using the telesupervised adaptive ocean sensor fleet system," Proc. International Conference on Robotics and Automation (ICRA), 1061-1068 (2008).

[4] Podnar, G.W., Dolan, J.M. and Elfes, A., "Networked architecture for robotic environmental ocean science sensors," Proc. Sensing a Changing World Workshop (2008).

[5] Low, K. H., Podnar, G.W., Stancliff, S., Dolan, J.M. and Elfes, A., "Robot Boats as a Mobile Aquatic Sensor Network," Proc. Workshop on Sensor Networks for Earth and Space Science Applications (ESSA) at the International Conference on Information Processing in Sensor Networks (2009).

[6] Elfes, A., "Multi-source spatial fusion using Bayesian reasoning," [Data Fusion in Robotics and Machine Intelligence], Academic Press (1992).

[7] Elfes, A., "Robot navigation: Integrating perception, environmental constraints and task execution within a probabilistic framework," [Reasoning with Uncertainty in Robotics], Springer Verlag, Berlin, 91-130, (1996).

[8] Low, K.H., Dolan, J.M. and Khosla, P.K., "Adaptive multi-robot wide-area exploration and mapping," Proc. Conference on Autonomous Agents and Multi-Agent Systems (AAMAS), 23-30 (2008). 